\title{
Atención dental asociada con un brote de infección por VIH en pacientes en diálisis ${ }^{1}$
}

\author{
Leonelo E. Bautista ${ }^{2}$ y Myriam Oróstegui ${ }^{3}$
}

RESUMEN En mayo de 1993 se descubrió fortuitamente un brote de 14 casos de infección por virus de la inmunodeficiencia humana (VIH) en pacientes sometidos a hemodiálisis en un hospital universitario de Bucaramanga, Colombia. El brote tuvo lugar en 1992. Se usaron sueros almacenados para determinar el período de infección probable (PIP) de 10 de los 14 casos y se llevó a cabo un estudio de casos y controles anidado a fin de evaluar los posibles mecanismos de transmisión. La atención de salud recibida por cada paciente positivo a VIH durante su PIP se comparó con la atención recibida por controles apareados con los casos en tiempo. Solamente los procedimientos odontológicos invasores mostraron una asociación significativa con el riesgo de infección. Los pacientes sometidos a procedimientos dentales invasores durante su PIP tuvieron un riesgo promedio de infección 8,15 veces mayor que los controles de características similares $(\mathrm{P}=0,006), y$ de nueve casos de infección por VIH con PIP conocidos en 1992, siete habian sido sometidos a un procedimiento dental invasor 1 a 6 meses antes de la seroconversión. No se detectó infección en ninguno de los miembros del personal de salud dental. A juzgar por las pruebas disponibles, lo más probable es que la infección haya sido transmitida de un paciente a otro por instrumentos dentales contaminados.

La transmisión del virus de la inmunodeficiencia humana (VIH) durante la atención de la salud es rara. El riesgo de que ocurra transmisión de un paciente infectado a un trabajador de salud por exposición percutánea a sangre infectada es de aproximadamente tres oportunidades en 1000 (1). El riesgo de transmisión de un trabaja-

\footnotetext{
1 Se publicó en inglés en la Revista Panamericana de Salud Pública/Pan American Journal of Public Health, Vol. 2, No. 3, 1997 con el título "Dental care associated with an outbreak of HIV infection among dialysis patients".

2 Universidad Industrial de Santander, Escuela de Medicina, Bucaramanga, Colombia. Dirección postal: Universidad Industrial de Santander, Facultad de Salud, Departamento de Salud Pública, Carrera 32, No. 29-31, 3er. piso, Bucaramanga, Colombia.

3 Universidad Industrial de Santander, Escuela de Medicina, Bucaramanga, Colombia.
}

dor de salud a un paciente debería ser aún más bajo, pero nunca ha sido documentado adecuadamente (2). No obstante, a menudo se han descrito casos de transmisión de un paciente a otro por medio de equipo contaminado $(3,4)$ y la reutilización de agujas contaminadas en el África se ha considerado un importante mecanismo de transmisión del VIH (5).

Son pocos los brotes de infección por VIH que se han vinculado con la atención médica. No obstante, los Centros para el Control y la Prevención de Enfermedades (CDC) de los Estados Unidos de América notificaron en 1990 la existencia de un conglomerado de pacientes odontológicos infectados por VIH $(6,7)$. Se descubrió en ese caso que los pacientes se habían infectado durante la atención dental y se conje- turaron tres posibles vías de transmisión: de paciente a paciente mediante equipo contaminado; de un dentista infectado al paciente por cortes accidentales (penetración de sangre del dentista en la boca ensangrentada de los pacientes); y transmisión intencionada del dentista infectado a los pacientes por un mecanismo desconocido $(8,9)$. Pese a que los CDC prefirieron la hipótesis de que hubo transmisión no intencionada del dentista a los pacientes (7), otros autores (10-12) opinaron que el virus podría haberse transmitido de un paciente a otro. Lamentablemente, se carecía de pruebas fehacientes que apoyaran cualquiera de estas hipótesis (9).

En otros lugares, una serie de casos de infección por VIH transmitida por contagio intrainstitucional dentro de 
un hospital pediátrico en Rusia aparentemente se debió a la reutilización de agujas no esterilizadas (3). En 1993 también se notificaron en la Argentina dos series de casos de infección por VIH entre pacientes en hemodiálisis (13). Aunque ninguno de los dos brotes en la Argentina fue estudiado oficialmente ni descrito en la literatura publicada, la reutilización de los filtros de diálisis para distintos pacientes parece haber sido el mecanismo de transmisión más probable.

En el trabajo aquí descrito hemos estudiado un brote de casos de infección por VIH en pacientes crónicos en hemodiálisis dentro de un hospital universitario de Bucaramanga, Colombia, con el propósito de identificar los posibles mecanismos de transmisión. El brote se produjo en 1992 y fue descubierto de forma fortuita en mayo de 1993. Otro estudio sobre este brote fue publicado anteriormente (14). En el presente artículo se examina la validez de los resultados de ese estudio.

\section{MATERIALES Y MÉTODOS}

\section{Búsqueda de casos}

Se buscaron casos entre todos los candidatos a trasplantes de riñón que habían utilizado la unidad de hemodiálisis a partir de 1982. Como parte del programa de trasplante de riñón, cada mes se habían tomado muestras de sangre de estos pacientes y se habían almacenado muestras de suero para realizar ensayos de inmunocompatibilidad. Los sueros almacenados y las muestras de sangre de que se disponía fueron sometidos a ensayos para detectar la presencia de anticuerpos contra VIH mediante la prueba de inmunoabsorción enzimática (ELISA). Todos los casos con resultados positivos en la ELISA fueron confirmados mediante la prueba de inmunoelectrotransferencia o western blot, salvo en un solo caso cuyo resultado fue impreciso.

Para determinar el momento de la seroconversión se aplicaron las pruebas a los sueros almacenados de cada paciente, comenzando con el suero más reciente y continuando hacia el más antiguo, hasta que una muestra diera resultados negativos a VIH. El período de infección probable (PIP) de cada paciente infectado se definió como el intervalo entre la fecha del suero negativo a VIH más reciente del paciente y la fecha del primer suero positivo a VIH.

También buscamos casos de VIH entre el personal sanitario encargado de atender a los pacientes en diálisis, entre los pacientes de hemofilia que utilizaban el banco de sangre del hospital, entre las personas que habían donado la sangre recibida por los pacientes infectados (siempre que una o más de estas donaciones se hubiera hecho en el período comprendido entre los 3 meses anteriores al inicio del PIP y el final de ese PIP), y entre las parejas sexuales de los pacientes infectados.

\section{Diseño del estudio}

Los factores de riesgo que se estudiaron incluyeron datos sobre edad, sexo, estado civil, antecedentes de enfermedades de transmisión sexual (ETS), frecuencia de las diálisis, procedimientos invasores (2) (transfusión de sangre, trasplante de riñón, cirugía, cistoscopia, gastroscopia, punción torácica o lumbar y procedimientos odontológicos) de cada paciente. Los efectos de los factores de riesgo que no cambiaron durante el brote (sexo, edad, estado civil y antecedentes de ETS) fueron evaluados por medio de un estudio retrospectivo de cohortes en que se incluyó a todos los usuarios de la unidad de diálisis desde enero de 1990 hasta diciembre de 1993. Se calcularon los riesgos relativos en bruto y ajustados por el coeficiente de MantelHaenszel (15).

A fin de explorar los efectos de las múltiples diálisis, transfusiones y procedimientos invasores, realizamos un estudio anidado de casos y controles con un muestreo basado en densidades de incidencia (16). El método de muestreo permitió seleccionar del historial clínico de cada paciente uno o más períodos no repetidos y cada período seleccionado fue tratado como una unidad de observación separada durante el análisis. Los períodos comprendidos dentro del PIP y los anteriores al PIP se denominaron "período-caso" y "período-control", respectivamente.

Los "períodos-caso" provinieron solo de los pacientes infectados, mientras que los "períodos-control" provinieron tanto de pacientes infectados (antes de su PIP) como de pacientes no infectados. Todos los "períodos-caso" para los cuales se disponía de por lo menos un "período-control" de la misma duración y comprendido dentro de las mismas fechas fueron incluidos en el estudio. En vista de que todos los pacientes sometidos a diálisis durante el período de brote de mediados de 1992 resultaron infectados por VIH (excepto uno), la utilización de los "períodos-caso" y "períodos-control" fue necesaria para permitir el análisis de los datos.

La duración y fechas de los "períodos-caso" fue definida en relación con los "períodos-control" disponibles. Todos los "períodos-control" correspondientes a un "período-caso" se incluyeron en una serie correlacionada. Por ejemplo (cuadro 1), el paciente 12 contribuyó dos series apareadas: su experiencia durante mayo de 1992 se pudo aparear con la experiencia de los pacientes 2 y 5 durante el mismo mes; y su experiencia durante todo su PIP (desde comienzos de mayo hasta mediados de junio de 1992) se apareó con la experiencia del paciente 3 durante la misma época cronológica (figura 1).

Los "períodos-caso" se seleccionaron dentro del PIP de cada paciente infectado debido a que los acontecimientos que se produjeron durante el PIP eran los que más probablemente se asociaban con la adquisición de la infección. Por consiguiente, los acontecimientos comprendidos dentro los "períodos-caso" representaban una exposición etiológicamente pertinente (17). En cambio, los "períodoscontrol" fueron seleccionados de la fase anterior al comienzo del PIP y solo los acontecimientos que se produjeron durante un período de igual duración que el "período-caso" del 
CUADRO 1. Fecha de detección de la infección por VIH y duración del período de infección probable (PIP) de cada caso estudiado, 1990 a 1993

\begin{tabular}{|c|c|c|c|}
\hline $\begin{array}{l}\text { Número } \\
\text { del caso }\end{array}$ & $\begin{array}{c}\text { Fecha de la } \\
\text { última prueba } \\
\text { negativa a VIH }\end{array}$ & $\begin{array}{c}\text { Fecha de la } \\
\text { primera prueba } \\
\text { positiva a VIH }\end{array}$ & $\begin{array}{l}\text { Duración } \\
\text { del PIP } \\
\text { (meses) }\end{array}$ \\
\hline 8 & 25 de enero de 1990 & 15 de mayo de 1990 & 3,6 \\
\hline 12 & 12 de mayo de 1992 & 23 de junio de 1992 & 1,4 \\
\hline 1 & 2 de julio de 1991 & 3 de julio de 1992 & 12,0 \\
\hline 2 & 4 de junio de 1992 & 9 de julio de 1992 & 1,1 \\
\hline 15 & 4 de mayo de 1992 & 27 de julio de 1992 & 2,8 \\
\hline 14 & 27 de marzo de 1992 & 2 de agosto de 1992 & 4,2 \\
\hline 6 & 2 de septiembre de 1991 & 4 de agosto de 1992 & 11,0 \\
\hline 3 & 7 de julio de 1992 & 5 de agosto de 1992 & 1,0 \\
\hline 5 & 4 de junio de 1992 & 10 de agosto de 1992 & 2,2 \\
\hline 7 & 7 de febrero de 1992 & 2 de septiembre de 1992 & 6,8 \\
\hline $11^{\mathrm{a}}$ & - & 18 de mayo de 1992 & - \\
\hline $16^{a}$ & - & 17 de mayo de 1993 & - \\
\hline $17^{a}$ & - & 13 de mayo de 1993 & - \\
\hline $4^{a}$ & - & 12 de febrero de 1993 & - \\
\hline
\end{tabular}

${ }^{\text {a }}$ No se dispone de muestras de suero negativo a $\mathrm{VIH}$.

paciente y que estuviera comprendido entre las mismas fechas se consideraron exposiciones pertinentes.

Para el análisis de los datos se utilizó la regresión logística condicionada (18); no se excluyó ninguna de las variables estudiadas en el modelo de regresión debido a que todas se consideraron posibles factores de riesgo. En vista de que los períodos mencionados por un paciente podrían no ser independientes, utilizamos la regresión logística binomial aleatoria (19) para tener en cuenta esta posibilidad y obtener pruebas estadísticas e intervalos de confianza valederos. Se calcularon las estadísticas delta-beta de Pregibon para evaluar la influencia de cada observación sobre los resultados del estudio (20).

\section{RESULTADOS}

La unidad, que contaba con siete aparatos de diálisis, trataba diariamente a un promedio de 10 pacientes. Los pacientes seguían una de tres pautas de tratamiento: recibían diálisis los lunes, miércoles y viernes; los martes y viernes; o los lunes y jueves. Entre 1990 y 1993, la unidad atendió a 49 pacientes crónicos en diálisis. Solo 35 de ellos tenían por lo menos una muestra de suero almacenada o se encontraban disponibles para la toma de muestras de sangre cuando se detectó el brote de VIH. Todos los demás pacientes habían fallecido o

FIGURA 1. Resultados del seguimiento serológico de los pacientes sometidos a diálisis en el hospital donde se hizo el estudio desde abril de 1991 hasta agosto de 1993 inclusive

$1991 \quad 1992 \quad 1993$

\begin{tabular}{|c|}
\hline LEYENDA \\
Última prueba \\
negativa a VIH $\nabla$ \\
Primera prueba \\
positiva a VIH $\nabla$ \\
Última prueba \\
negativa a VHB $\nabla$ \\
Primera prueba \\
positiva a VHB \\
Inicio de la diálisis \\
aislada del paciente $\diamond$ \\
Período que \\
duró la diálisis \\
\hline
\end{tabular}

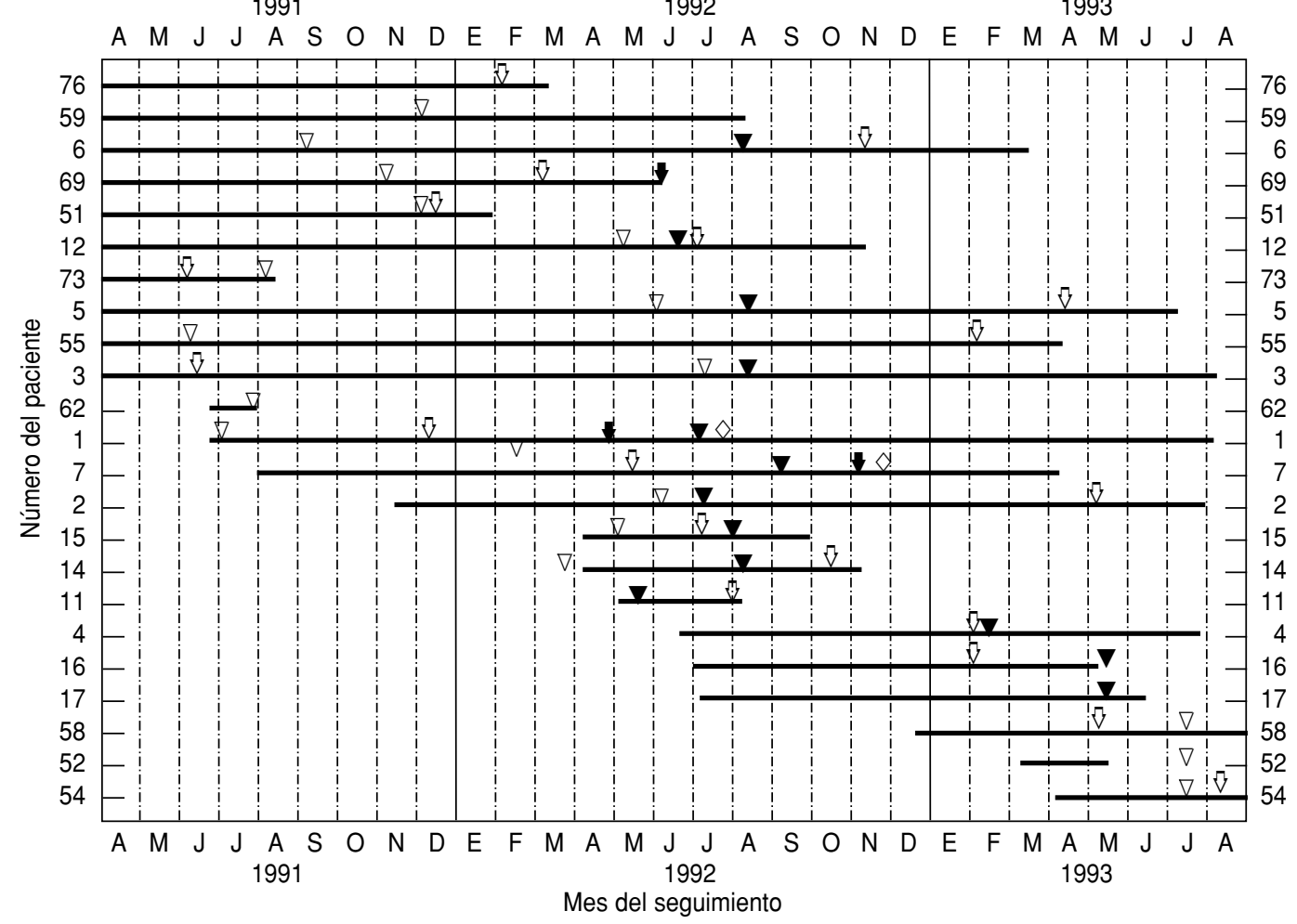


habían recibido un trasplante de riñón con buenos resultados. Cuando se detectó el brote, $14(40 \%)$ de los 35 pacientes con muestras de suero disponibles resultaron infectados por VIH.

Uno de los pacientes fue identificado como seropositivo por primera vez utilizando una muestra de 1990, 10 fueron identificados con muestras tomadas en 1992 y tres fueron identificados con muestras de 1993 (véase el cuadro 1). La duración del PIP se determinó en 10 casos y varió de uno a 12 meses, con una mediana de 3 meses. Es probable que la duración del PIP se haya sobreestimado en tres pacientes cuyos PIP duraron más de 6 meses, debido a que cada uno de estos pacientes tenía disponible solo una muestra de suero almacenada anterior a su primer suero positivo a VIH. La duración del PIP no pudo determinarse en el caso de cuatro pacientes por no contarse con muestras de suero anteriores a la primera muestra positiva a VIH.

De los nueve pacientes cuyos PIP pudieron definirse y que se sabía que habían ocurrido en 1992, tres tuvieron su primera prueba positiva a VIH en julio y cuatro en agosto de 1992 (cuadro 2 y figura 1). A fines de 1992, 10 pacientes tratados con diálisis durante ese año se habían infectado; un paciente que inició su diálisis en diciembre de 1992 no dio pruebas serológicas de infección y no se conocía el estado de los otros tres pacientes que fueron positivos a VIH en 1993. Un paciente comenzó su tratamiento de diálisis en marzo de 1993 y otro al mes siguiente. Cuando se detectó el brote (en mayo de 1993), ninguno de ellos estaba infectado (figura 1). A fines de 1993 , siete $(50 \%)$ de los 14 pacientes infectados y $13(61,9 \%)$ de los 21 pacientes en diálisis no infectados que figuran en el cuadro 3 habían fallecido. (Prueba exacta bilateral de Fisher, $P=0,511$.)

Durante el análisis retrospectivo de las muestras de suero se identificó a otro paciente infectado. Este paciente, que ya estaba infectado al entrar al programa de diálisis en 1988, fue excluido del análisis puesto que su infección se produjo demasiado tem-

CUADRO 2. Número de pacientes de diálisis en 1992 y riesgo de infección por VIH entre ellos durante ese año

\begin{tabular}{|c|c|c|c|c|c|c|c|}
\hline \multirow[b]{3}{*}{ Mes } & \multicolumn{2}{|c|}{ Pacientes } & \multirow{2}{*}{\multicolumn{2}{|c|}{ Estado en cuanto al VIH }} & \multirow{2}{*}{\multicolumn{2}{|c|}{ Casos en 1992}} & \multirow{3}{*}{$\begin{array}{c}\text { Riesgo } \\
\text { (porcentaje) }\end{array}$} \\
\hline & \multirow{2}{*}{$\begin{array}{c}\text { Número } \\
\text { total }\end{array}$} & \multirow{2}{*}{$\begin{array}{c}\text { En } \\
\text { peligro }\end{array}$} & & & & & \\
\hline & & & Positivo $^{a}$ & Desconocido & Nuevos & Acumulados $^{b}$ & \\
\hline Enero & 11 & 8 & $3^{c}$ & 0 & 0 & 0 & 0,0 \\
\hline Febrero & 10 & 8 & 2 & 0 & 0 & 0 & 0,0 \\
\hline Marzo & 10 & 8 & 2 & 0 & 0 & 0 & 0,0 \\
\hline Abril & 11 & 9 & 2 & 0 & 0 & 0 & 0,0 \\
\hline Mayo & 13 & 10 & 3 & 0 & 0 & 1 & 0,0 \\
\hline Junio & 13 & 9 & 3 & 1 & 1 & 2 & 11,1 \\
\hline Julio & 14 & 8 & 4 & 2 & 3 & 5 & 37,5 \\
\hline Agosto & 11 & 5 & 4 & 2 & 4 & 9 & 80,0 \\
\hline Septiembre & 11 & 1 & 3 & 7 & 1 & 10 & 100,0 \\
\hline Octubre & 10 & 1 & 3 & 6 & 0 & 10 & 0,0 \\
\hline Noviembre & 11 & 1 & 3 & 7 & 0 & 10 & 0,0 \\
\hline Diciembre & 9 & 1 & 3 & 5 & 0 & 10 & 0,0 \\
\hline
\end{tabular}

a Continúa en diálisis.

b Acumulados durante 1992

c Un paciente fue infectado en 1988 y dos en 1990.

prano como para estar vinculada al brote.

El paciente 11 proporcionó la muestra de suero positiva a VIH más antigua al haberse infectado con VIH en 1992. La muestra positiva fue tomada 2 semanas después del comienzo de la hemodiálisis del paciente. El paciente fue sometido a un solo procedimiento odontológico invasor que se produjo 6 meses después de comenzar la hemodiálisis, cuando ya era positivo a VIH.

Cuando se detectó el brote en mayo de 1993, cinco de los pacientes infectados ya habían fallecido. Tres de los nueve sobrevivientes informaron que no tenían pareja sexual estable, mientras que los seis restantes dijeron tenerla. Solo una de estas parejas sexuales resultó positiva a VIH. No se detectaron otros casos de infección por VIH entre 100 trabajadores de la salud sometidos a la prueba o entre los 26 pacientes de hemofilia que utilizaban periódicamente el banco de sangre del hospital. De las 56 personas que habían donado sangre a los pacientes infectados, 21 fueron localizadas pero ninguna resultó positiva a VIH.

Quince $(42,9 \%)$ de los 35 pacientes originales recibieron un trasplante de riñón antes de 1993 o en el transcurso del año. Pese a que dos de los seis pacientes positivos a VIH que recibieron trasplantes eran negativos a VIH antes de ellos, los donantes de sus riñones también resultaron seronegativos a VIH.

Los análisis en bruto y ajustados del estudio de cohorte retrospectivo demostraron que el riesgo de infección no estaba asociado significativamente con la edad, el sexo o el estado civil (valores $P$ de la prueba exacta bilateral de Fisher: sexo: 0,09; edad: 0,95, y estado civil: 0,40; véanse los datos del cuadro 3). En cambio, todos los cuatro pacientes con antecedentes de ETS resultaron positivos a $\mathrm{VIH}$, frente a solo $10(32,2 \%)$ de los 21 pacientes sin antecedentes de ETS (prueba exacta bilateral de Fisher: $P=0,02$ ).

El estudio de casos y controles anidado comprendió datos de pacientes infectados con PIP conocidos y de un paciente no infectado. Uno o más de los "períodos-control" fueron identificados para todos los "períodos-caso", excepto para los "períodos-caso" aportados por el paciente 3 (véase la figura 1); en total, comparamos 34 "períodoscaso" proporcionados por pacientes infectados $(12,1,2,15,14,6,5$ y 7; véase el cuadro 1) con 51 "períodoscontrol" suministrados por el paciente no infectado y otros 10 pacientes antes del comienzo de sus PIP $(59,6,69,51$, $12,5,3,62,7,2$ y 15; véase la figura 1).

Los pacientes que habían tenido endoscopia $u$ otros procedimientos invasores (además de tratamientos 
CUADRO 3. Riesgo de infección por VIH, desglosado por sexo y edad, de los pacientes en diálisis del hospital en que se realizó el estudio, 1990-1993

\begin{tabular}{|c|c|c|c|c|c|c|c|c|c|}
\hline \multirow[b]{2}{*}{$\begin{array}{l}\text { Edad } \\
\text { (años) }\end{array}$} & \multicolumn{3}{|c|}{ Hombres } & \multicolumn{3}{|c|}{ Mujeres } & \multicolumn{3}{|c|}{ Total } \\
\hline & $\begin{array}{l}\text { Número de } \\
\text { pacientes }\end{array}$ & $\begin{array}{l}\text { Número de } \\
\text { casos de VIH }\end{array}$ & $\begin{array}{c}\text { Riesgo } \\
\text { (porcentaje) }\end{array}$ & $\begin{array}{l}\text { Número de } \\
\text { pacientes }\end{array}$ & $\begin{array}{l}\text { Número de } \\
\text { casos de VIH }\end{array}$ & $\begin{array}{c}\text { Riesgo } \\
\text { (porcentaje) }\end{array}$ & $\begin{array}{l}\text { Número de } \\
\text { pacientes }\end{array}$ & $\begin{array}{c}\text { Número de } \\
\text { casos de VIH }\end{array}$ & $\begin{array}{c}\text { Riesgo } \\
\text { (porcentaje) }\end{array}$ \\
\hline $20-29$ & 3 & 2 & 66,7 & 2 & 0 & 0,0 & 5 & 2 & 40,0 \\
\hline 30-39 & 6 & 3 & 50,0 & 5 & 2 & 40,0 & 11 & 5 & 45,4 \\
\hline $40-49$ & 10 & 5 & 50,0 & 3 & 0 & 0,0 & 13 & 5 & 38,5 \\
\hline
\end{tabular}

Valores de la prueba exacta bilateral de Fischer: sexo $=0,09 ;$ edad $=0,97$; estado civil $=0.40 ;$ antecedentes de enfermedades de transmisión sexual $=0,02$.

dentales) no demostraron correr un riesgo mayor de infección por $\mathrm{VIH}$ aun después del ajuste para corregir el efecto de otras variables (cuadro 4). Tampoco la transfusión de sangre o la hemodiálisis estaban asociados con un riesgo de infección por VIH significativamente mayor. Otros posibles factores de riesgo, tales como la cirugía, eran demasiado infrecuentes entre las personas afectadas como para haber servido de vía principal de transmisión.

De todos los factores de riesgo estudiados, solamente los procedimientos dentales invasores (PDI) estaban asociados significativamente con la infección por VIH, tanto en los análisis en bruto como en los ajustados (véase el cuadro 4). De hecho, el riesgo ajustado de infección por VIH era 8,15 veces más elevado en los pacientes que tuvieron procedimientos dentales invasores durante su PIP que en los que no los tuvieron (intervalo de con- fianza de 95\% (IC95\%): 1,85 a 36,0); y subió a casi el triple por cada procedimiento odontológico invasor (razón de posibilidades: 2,98; IC95\%: 1,21 a 7,36). Los resultados no cambiaron con un análisis de regresión logística basada en efectos al azar (el intercepto aleatorio no resultó estadísticamente significativo, $P=0,11)$.

El número promedio de procedimientos odontológicos invasores en los pacientes del estudio fue de 1,73 durante los "períodos-caso" y 0,36 durante los "períodos-control". De los seis pacientes que contribuyeron al "período-caso", cinco fueron sometidos a procedimientos odontológicos invasores durante su PIP, mientras que solo dos de los 11 que aportaron "períodos-control" habían sufrido estos procedimientos durante esos períodos (prueba exacta bilateral de Fisher, $P=0,07)$. De los 10 pacientes positivos a VIH con PIP conocidos, el único caso que resultó positivo en las pruebas de 1990 nunca tuvo un procedimiento odontológico invasor y probablemente no tuvo relación con el brote de 1992. No obstante, ocho de los nueve pacientes que resultaron positivos a VIH por primera vez en 1992 habían experimentado un procedimiento odontológico invasor uno a 6 meses antes de la seroconversión (en siete casos) o 21 meses antes de la seroconversión (en el octavo caso). En este grupo, la mediana del tiempo transcurrido entre el procedimiento odontológico y la primera muestra positiva a VIH fue de 3,5 meses. No se determinó ningún procedimiento odontológico invasor en uno de los nueve pacientes que resultaron seropositivos en 1992.

Estudiamos las fechas en que los pacientes infectados recibieron procedimientos odontológicos invasores a fin de identificar las posibles vías de transmisión dentro de la unidad odon-

CUADRO 4. Razones de posibilidades brutas y ajustadas según los factores de riesgo de infección por VIH, 1992

\begin{tabular}{|c|c|c|c|c|c|c|}
\hline \multirow[b]{2}{*}{$\begin{array}{c}\text { Factor } \\
\text { de riesgo }\end{array}$} & \multicolumn{3}{|c|}{ Razón de posibilidades (cifras brutas) } & \multicolumn{3}{|c|}{ Razón de posibilidades (cifras ajustadas)a } \\
\hline & $\begin{array}{c}\text { Razón de } \\
\text { posibilidades }\end{array}$ & IC95\% & $P^{c}$ & $\begin{array}{l}\text { Índice de } \\
\text { riesgo }\end{array}$ & IC95\% & $P^{c}$ \\
\hline Cistoscopia & 2,11 & $0.46-9.79$ & 0,34 & 1,47 & $0.23-9.26$ & 0,68 \\
\hline Gastroscopia & 2,12 & $0.68-6.62$ & 0,20 & 1,21 & $0.25-9.26$ & 0,61 \\
\hline \multicolumn{7}{|l|}{ Otros procedimientos odontológicos } \\
\hline no invasores ${ }^{d}$ & 2,16 & $0.20-23.88$ & 0,53 & 1,36 & $0.10-17.98$ & 0,62 \\
\hline Transfusiones de sangre & 1,05 & $0.35-3.15$ & 0,94 & 1,31 & $0.35-4.94$ & 0,69 \\
\hline Hemodiálisis & 1,01 & 0.99-1.02 & 0,52 & 0,99 & $0.96-1.01$ & 0,36 \\
\hline Procedimientos odontológicos invasores & 6,36 & 1.97-20.51 & 0,002 & 8,15 & $1.85-36.0$ & 0,006 \\
\hline
\end{tabular}

a Ajustadas según las variables que figuran en el cuadro.

b IC = Intervalo de confianza

${ }^{c}$ Valor $P$ de la prueba de Wald.

${ }^{\mathrm{d}}$ Comprende las punciones torácica y lumbar. 
tológica. Solo encontramos tres pacientes que habían recibido atención dental el mismo día (todos antes de su primera prueba positiva a VIH) y otros dos que fueron atendidos con 2 días de diferencia (uno sin infectar y otro con estado desconocido con respecto al VIH). Estos datos no permitieron identificar una cadena de transmisión.

Los datos preliminares del estudio del genoma del VIH indicarían que la secuencia de nucleótidos provenientes de regiones variables y constantes de la glucoproteína gp 120 era más semejante entre virus tomados de los pacientes infectados en este brote que entre virus provenientes del grupo control (21).

\section{DISCUSIÓN}

Nuestros resultados indican que los procedimientos odontológicos invasores proporcionaron el mecanismo más probable para la transmisión de VIH durante este brote. Si bien los antecedentes de ETS mostraron una fuerte asociación con un mayor riesgo de infección por VIH, ninguno de los pacientes infectados tuvo una enfermedad de transmisión sexual durante 1992, ninguno contaba con más de una pareja sexual, y solo una de las seis parejas sexuales conocidas resultó positiva al VIH. Estos datos sugieren que la asociación observada entre ETS y la infección por VIH era fortuita.

Tampoco es probable que transfusiones de sangre contaminada por VIH fueran la fuente de la infección. Los bancos de sangre de la ciudad han practicado pruebas de detección de VIH a partir de 1988; el número de transfusiones fue similar en los períodos "caso" y "control" y no se encontraron personas infectadas entre las que donaron la sangre recibida por los pacientes infectados o por los pacientes hemofílicos que acudían periódicamente al banco de sangre del hospital.

El número de diálisis recibido por los pacientes del estudio no mostró asociación con el riesgo de infección por VIH. Si bien otros autores (14) han afirmado que este brote fue causado por intercambio de agujas de acceso contaminadas, no encontramos pruebas en apoyo de esa hipótesis. De hecho, estos resultados son cuestionables porque los autores compararon pacientes que utilizaron la unidad de diálisis durante el brote con pacientes que la utilizaron antes del brote. Asimismo, definieron la exposición según el estado del paciente en relación con la infección por VIH y sin ofrecer pruebas de una vinculación entre el intercambio de las agujas y el riesgo de infección. Como las agujas se almacenaban en bandejas rotuladas con los nombres de los pacientes y cada lunes se utilizaban agujas nuevas, es improbable que se intercambiaran las agujas. Tampoco es probable que el VIH pudiera sobrevivir en las agujas utilizadas para la diálisis porque estas se sumergían en una solución de cloruro de benzalconio al $0,16 \%$ durante un mínimo de 48 horas $(22,23)$.

Como los filtros y las líneas de diálisis se usaban de forma individual, se limpiaban a fondo con solución salina después de usados y se llenaban con una solución de formol al 5\% durante 24 a 48 horas antes de volver a darles uso, parecería improbable la transmisión por este medio. La transmisión por las propias máquinas de diálisis es imposible porque esos aparatos no entran en contacto directo con la sangre del paciente.

Por añadidura, en 1988 y 1990 se hicieron diálisis periódicas a dos pacientes infectados por VIH, sin que se tuviera conocimiento de su estado con respecto al VIH. Aunque el personal y los procedimientos de manejo del equipo fueron los mismos que en 1992, aparentemente no hubo brotes de VIH durante estos años. Esto apoya la teoría de que la diálisis no fue la responsable de la transmisión del VIH.

Tampoco los antecedentes de infección por virus de la hepatitis B (VHB) de los pacientes estudiados sugieren que la transmisión se haya producido dentro de la unidad de diálisis. Se hicieron pruebas trimestrales de hepatitis B a los pacientes. Los pacientes $1 \mathrm{y}$ 7 se infectaron con VHB en 1992 y por lo tanto empezaron su diálisis con un equipo separado. En esa época ya eran positivos a VIH (figura 1). Pese a que el paciente 69 también era positivo al VHB, solo fue identificado como tal por una muestra de sangre tomada un día después de su última diálisis. En total estos tres pacientes recibieron por lo menos 47 diálisis en equipo compartido con otros pacientes después de convertirse en positivos al VHB. Si bien el VHB es un agente mucho más infeccioso que el VIH $(2,24)$, solo tres de los 14 pacientes se infectaron con el $\mathrm{VHB}$, frente a nueve de los 10 infectados con VIH. Por consiguiente, la ausencia de un brote de VHB indica que la diálisis no fue el vehículo responsable de la transmisión mucho más generalizada del VIH entre los pacientes estudiados.

Si bien muchos procedimientos invasores representan posibles vías de transmisión del VIH (2), ninguno de ellos, salvo los procedimientos odontológicos, se mostraron asociados significativamente con el riesgo de infección por VIH. Algunos datos adicionales apoyan la transmisión odontológica: los pacientes 52, 54 y 58 (figura 1) y cuatro pacientes crónicos ambulatorios de diálisis peritoneal fueron los únicos candidatos a trasplante de riñón en 1992 que no se infectaron con VIH. Estos pacientes nunca recibieron un tratamiento odontológico invasor. También el paciente 14 (que nunca reutilizó el equipo de diálisis) y el paciente 10 (que nunca recibió diálisis en esta unidad) fueron tratados con un procedimiento odontológico invasor 2 a 4 meses antes de su seroconversión en positivos a VIH. Por último, todos salvo uno de los pacientes infectados en 1992 fueron sometidos a procedimientos odontológicos invasores antes de su seroconversión. Este único paciente, también con seroconversión en 1992, no tenía antecedentes de ETS. En vista de que a todos los candidatos a trasplante de riñón se les hacía por lo menos un examen dental, la ausencia de todo el informe odontológico de este paciente sugiere la pérdida de una parte de su registro clínico. 
El elevado riesgo de infección y la brevedad de los PIP sugieren que el VIH se transmitió por vía sanguínea. Dado que ninguno de los trabajadores de la atención odontológica resultó infectado y que los pacientes infectados parecían tener la misma cepa del VIH, la transmisión de paciente a paciente por medio de instrumentos odontológicos contaminados constituye la forma de contagio más probable. No obstante, la superposición de los PIP no permitió identificar un caso índice. En vista de que el servicio odontológico atendía a otros pacientes, no se puede descartar la posibilidad de que el caso índice haya sido un paciente ajeno al grupo de diálisis.

La ausencia de una cadena definida de transmisión dentro de la unidad odontológica puede deberse a varias circunstancias. En primer lugar, la verdadera fecha de infección de un paciente depende de cuándo este adquirió el VIH y no de cuando proporcionó la muestra de sangre almacenada en que se observó el resultado positivo a VIH. Esto impide definir con certeza cualquier secuencia progresiva de transmisiones de VIH. En segundo lugar, no se contaba con los sueros almacenados de los pacientes que habían fallecido o recibido un trasplante de riñón con buenos resultados. En tercer lugar, no podemos desestimar la posibilidad de que otros pacientes odontológicos (tales como los pacientes internados en el hospital que no eran candidatos a un trasplante de riñón, o pacientes externos cuyos datos no estaban disponibles) hubieran sido vínculos en la cadena de la transmisión.

La transmisión del VIH por instrumentos odontológicos contaminados es una posibilidad porque el VIH se encuentra en la sangre, saliva (25) y pulpa dentaria (26) de los pacientes infectados. Hacia fines de 1991, el servicio odontológico comenzó a atender a pacientes positivos a VIH enviados por la Liga contra el Sida y ello puede haber provocado el brote de infección por VIH entre los pacientes en diálisis. En septiembre de 1992 el personal odontológico se negó a atender a pacientes infectados por VIH debido a que el servicio de odontología carecía de los recursos apropiados.

Es poco probable que nuestros resultados estén sesgados. Solamente se excluyó del estudio a los pacientes con PIP desconocido y parecería improbable que el hecho de no disponer en estos casos de sueros almacenados guarde relación con haber pasado por procedimientos odontológicos invasores o con la presencia o no de infección por VIH. Los factores de confusión fueron controlados mediante regresión múltiple y la obtención de "períodoscaso" y "períodos-control" de los mismos pacientes. Otros posibles factores de riesgo - tales como la utilización de drogas inyectables, el tatuaje y otros procedimientos con penetración de la piel- eran infrecuentes en la población estudiada y no fueron mencionados por los sobrevivientes entrevistados. Ninguno de los pacientes varones admitió haber tenido contactos homosexuales. En vista de que las fichas clínicas de los pacientes del estudio se completaron sin tener conocimiento de su positividad a VIH, los errores del registro no deberían ser de carácter diferencial y en todo caso habrían atenuado la asociación entre los procedimientos odontológicos invasores y la infección por VIH (27).

Se puede afirmar justificadamente que si los pacientes infectados por el VHB hubieran sido sometidos a procedimientos odontológicos invasores, se habría constatado una mayor tasa de infección por el VHB entre los pacientes en diálisis. Sin embargo, a excepción de un paciente infectado por el VHB que había sufrido tres procedimientos odontológicos invasores, ningún otro paciente infectado por el VHB fue sometido a ningún procedimiento odontológico invasor durante el período de duración del brote. Por lo tanto, es poco probable que la transmisión del VHB se deba a los procedimientos odontológicos invasores.

Por el hecho de haber considerado etiológicamente pertinente solo la exposición durante el PIP, podríamos haber omitido acontecimientos importantes anteriores a la última prueba con resultado negativo a VIH, siempre que dicho resultado negativo hubiera sido falso. Esto podría haber ocurrido si el paciente se encontraba en la fase de la ventana inmunológica. Sin embargo, en vista de la alta sensibilidad de la ELISA al VIH, la posibilidad de un resultado negativo falso era muy pequeña. En todo caso, los errores debidos a pruebas falsas negativas no deberían ser diferenciales y habrían diluido el efecto estimado de los procedimientos odontológicos invasores. Asimismo, como los registros clínicos se habían completado sin tener conocimiento del estado del paciente con respecto a la presencia o no de la infección por $\mathrm{VIH}$, los errores de registro no hubieran sido diferenciales y de hecho podrían haber atenuado la asociación entre el procedimiento odontológico invasor y la infección por $\operatorname{VIH}(17,27)$. Aunque no contamos con pruebas directas, el VIH podría haberse transmitido a los pacientes estudiados por medio del instrumental manual o de ángulos de profilaxis contaminados (piezas manuales de baja velocidad que se utilizan para la limpieza y el pulido de los dientes) (28), la utilización incorrecta del eyector de saliva (29), o instrumentos quirúrgicos indebidamente esterilizados. Ciertos estudios experimentales $(28,30-33)$ han demostrado que las turbinas y los tubos de agua en las piezas manuales y en los ángulos de profilaxis pueden contaminarse con líquidos orales del paciente durante su uso corriente. Esta contaminación podría ser particularmente grave en unidades odontológicas donde no hay válvulas de antirretracción o donde estas son defectuosas $(28,34,35)$. Lewis et al. (28) han recuperado partículas infecciosas de un bacteriófago, VIH y ADN del VHB del interior de las piezas y ángulos de profilaxis utilizados en pacientes infectados. Por consiguiente, parece obvio que si estos instrumentos no son tratados adecuadamente, los microorganismos de un paciente pueden llegar a la boca del siguiente.

Actualmente se recomienda la esterilización de las piezas manuales en autoclave entre un paciente y otro (36, 
37); pero la mayoría de las piezas manuales y ángulos de profilaxis no pueden resistir esas temperaturas ni pueden tratarse con desinfectantes internos y externos concentrados $(30,35$, 36). Por consiguiente, su tratamiento consiste principalmente en una limpieza externa con una solución desinfectante. Como la limpieza externa no resuelve el problema de la contaminación de las tuberías y turbinas $(30,32$, 35) los Centros para el Control y la Prevención de Enfermedades (CDC) de los Estados Unidos recomiendan hacer funcionar la pieza y descagar el agua en un vertedero por 20 ó 30 segundos entre distintos pacientes. Sin embargo, hay ciertas pruebas de que un lavado de esta duración recomendada podría ser demasiado breve (30).

El uso incorrecto del eyector de saliva también puede tener por resultado infecciones cruzadas (29). Puede producirse reflujo de líquidos hacia la boca del paciente cuando los labios del paciente se cierran alrededor de la punta del eyector. En ese caso, no basta con cambiar el eyector de saliva para prevenir una contaminación cruzada entre pacientes (29).

Cuando se produjo el brote que aquí se estudia, el servicio odontológico no tenía normas escritas para combatir las infecciones. Se utilizaba el calor seco para esterilizar el equipo odontológico; pero los hornos de esterilización no tenían termómetros y no se aplicaban métodos biológicos (para determinar la presencia de esporas). Además, los equipos odontológicos no tenían válvulas para impedir el reflujo; las piezas manuales y los ángulos de profilaxis no eran esterilizables y el eyector de saliva, que no era descartable, se limpiaba entre pacientes con una solución alcohólica.
En junio de 1990, los CDC $(6,7)$ comunicaron el primer caso de infección por VIH asociado con la atención odontológica. No se logró determinar el mecanismo de transmisión $(8,9)$, pero a pesar de ello varios investigadores (10-12) opinan que el virus podría haberse transmitido de un paciente a otro por equipo contaminado. No obstante la posibilidad de contagio con enfermedades de transmisión hemática durante la atención odontológica, no hay estudios publicados sobre el tema. Esto probablemente se deba a tres factores: los estudios sobre brotes de VIH asociados con la atención odontológica se han centrado en la transmisión del dentista al paciente $(2,30)$; no hay sistemas de detección eficientes para descubrir y notificar las infecciones cruzadas asociadas con la atención odontológica; y las infecciones por VIH y VHB se han atribuido tradicionalmente a los factores de riesgo más conocidos $(30,32)$.

Tras la detección del brote aquí descrito entraron en vigor medidas de lucha contra la infección en todos los servicios hospitalarios. Se suspendió la reutilización de las agujas y de los filtros de diálisis, así como el programa de trasplantes de riñón. Debido a la suspensión del programa, los candidatos a un trasplante de riñón ya no tenían que someterse a procedimientos odontológicos para erradicar las posibles fuentes de infección. Pese a que no hubo nuevas infecciones entre los pacientes de diálisis después de mayo de 1993, no pudimos desestimar la posibilidad de que hubiera otros casos entre los demás pacientes hospitalarios que no tenían sueros almacenados. El riesgo de infección entre estos últimos pacientes podría haber sido menor que el riesgo entre los candidatos al trasplante de riñón, que se someten a todo tipo de cuidados odontológicos necesarios para erradicar las posibles fuentes de infección. Esa atención odontológica minuciosa no es la habitual en los demás pacientes.

El estudio de los pacientes que concurrieron a la unidad odontológica en 1992 podría haber ayudado a aclarar nuestros resultados. Sin embargo, los registros de atención dental eran muy incompletos. Según los registros de la unidad de odontología, solo uno de los pacientes vinculados al brote fue reconocido como paciente odontológico en 1992. Felizmente, los candidatos a trasplante de riñón tenían sus antecedentes de atención odontológica incluidos en sus fichas clínicas porque esta información era necesaria para decidir si el paciente estaba listo para recibir un trasplante. No se guardaban registros similares para los demás pacientes del hospital. El descubrimiento del brote mucho tiempo (un año) después de su aparición dificultó en particular la localización de los anteriores usuarios de la unidad odontológica. La única opción razonable para identificar a los usuarios posiblemente infectados de la unidad odontológica hubiera sido un llamamiento al público, procedimiento que las autoridades sanitarias locales no autorizaron.

Nuestros resultados apuntan a un modo de transmisión de VIH que no se ha descrito antes. Aun en los países desarrollados, el control de las infecciones cruzadas durante la atención odontológica se ha considerado deficiente $(19,30,32,38)$. Eso sugiere elocuentemente que en los países en desarrollo la atención dental podría estar asociada con la infección por VIH y otras infecciones virales cruzadas $y$ podría constituir una importante fuente de diseminación de enfermedades transmitidas por vía hematógena.

\section{REFERENCIAS}

1. Henderson DK, Fahey BJ, Willy M, et al. Risk for occupational transmission of human immunodeficiency virus type 1 (HIV-1) associated with clinical exposures: a prospective evaluation. Ann Intern Med 1990;113:740-746.

2. Centers for Disease Control. Recommendations for preventing transmission of Human
Immunodeficiency Virus and Hepatitis B Virus to patients during exposure-prone invasive procedures. MMWR 1991;40(RR-8):1-9.

3. Pokrovskii VV, Eramova II, Deulina MO, et al. [An intrahospital outbreak of HIV infection in Elista] (Ruso). Zh Mikrobiol Epidemiol Immunobiol 1990;4:17-23.
4. Patrascu IV, Dumitrescu O. The epidemic of human immunodeficiency virus infection in Romanian children. AIDS Res Hum Retroviruses 1993;9:99-104.

5. Quinn TC, Mann JM, Curran JW, Piot P. AIDS in Africa: an epidemiologic paradigm. Science 1986;234:955-963. 
6. Centers for Disease Control. Possible transmission of human immunodeficiency virus to a patient during an invasive dental procedure. MMWR 1990;39:489-493.

7. Ciesielski C, Marianos D, Ou CY, et al. Transmission of human immunodeficiency virus in a dental practice [ver los comentarios]. Ann Intern Med 1992;116:798-805.

8. Robinson $\mathrm{P}, \mathrm{Challacombe} \mathrm{S}$. Transmission of HIV in a dental practice: the facts. $\mathrm{Br}$ Dent $\mathrm{J}$ 1993;175:383-384.

9. Breo DL. The dental AIDS cases-Murder or an unsolvable mystery? JAMA 1993;270: 2732-2734.

10. Taylor F. The Risk of transmission of HIV from health care professionals to patient (carta). JAMA 1991;266:1935.

11. Barondes JA. The risk of transmission of HIV from health care professional to patient (carta). JAMA 1991;266:1936.

12. Stark AN. Cross-contamination with dental equipment [carta; comentario]. Lancet 1993; 341:180.

13. Dyer E. Argentinian doctors accused of spreading AIDS. Br Med J 1993;307:584.

14. Velandia M, Fridkin SK, Cardenas V, et al. Transmission of HIV in dialysis centre. Lancet 1995;345:1417-1422.

15. Mantel N, Haenszel W. Statistical aspects of the analysis of data from retrospective studies of disease. I Natl Cancer Inst 1959;22:719-748.

16. Checkoway H, Pearce N, Crawford-Brown DJ. Case control studies: anonymous research methods in occupational epidemiology. New York: Oxford University Press; 1989.

17. Rothman K. Induction and latent periods. Am J Epidemiol 1981;114:253-259.

18. Hosmer DW, Lemeshow S. Applied logistic regression. New York: John Wiley and Sons; 1989.
19. Statistics and Epidemiology Research Corporation. EGRET, program manual. Statistics and Epidemiology Research Corporation; 1990.

20. Pregibon D. Logistic regression diagnostics. Ann Stat 1981;9:705-724.

21. Rengito B, Boshell J, Essex M, et al. Epidemiología molecular de una infección por el virus de la inmunodeficiencia humana en un hospital universitario colombiano. En: Resúmenes del VII Congreso Panamericano de Infectología. Cartagena, Colombia, 26 a 31 de mayo de 1995. Cartagena: Asociación Panamericana de Infectología; 1995.

22. Resnick L, Veren K, Salahuddin S, Tondreau S, Markham PD. Stability and inactivation of HTLV-III/LAV under clinical and laboratory environments. JAMA 1986;255:1887-1891.

23. Sattar SA, Springthorpe VS. Survival and disinfectant inactivation of the human immunodeficiency virus: a critical review. Rev Inf Dis 1991;13:430-447.

24. Gerberding JL. Reducing occupational risk of HIV infection. Hosp Pract 1991;26:103-118.

25. Chamberland ME, Curran JW. Epidemiology and prevention of AIDS and HIV infection. En: Mandell GL, Douglas RG Jr, Bennett JE. Principles and practice of infectious diseases. $3 \mathrm{a}$ ed. New York: Churchill Livingstone; 1990: 1029-1046.

26. Glick M, Trope M, Pliskin ME. Detection of HIV in the dental pulp of a patient with AIDS. J Am Dent Assoc 1989;119:649-650.

27. Copeland KT, Checkoway H, McMichael AJ Holbrook RH. Bias due to misclassification in the estimation of relative risk. Am J Epidemiol 1977;105:488-495.

28. Lewis DL, Arens M, Appleton SS, et al. Crosscontamination potential with dental equipment [ver los comentarios]. Lancet 1992;340: 1252-1254.
29. Watson CM, Whitehouse RL. Possibility of cross-contamination between dental patients by means of the saliva ejector. J Am Dent Assoc 1993;124:77-80.

30. Lewis DL, Boe RK. Cross-infection risks associated with current procedures for using highspeed dental handpieces [ver los comentarios]. J Clin Microbiol 1992;30:401-406.

31. Mills SE, Kuehne JC, Bradley DV. Bacteriological analysis of high-speed handpiece turbines. J Am Dent Assoc 1993;124:59-62.

32. Lewis DL. Infection control in dental handpieces (carta). ASM News 1991:57:393.

33. Shpuntoff H, Shpuntoff RL. High-speed dental handpieces and spread of airborne infections [ver los comentarios]. N Y State Dent J 1993;59:21-23.

34. Singh B, Bagga R, Murphy RA, Punwani I. Contamination of dental unit cooling water with oral microorganisms and its prevention. J Am Dent Assoc 1984;109:712-716.

35. Miller $\mathrm{CH}$. Cleaning, sterilization and disinfection: basics of microbial killing for infection control. J Am Dent Assoc 1993;124:48-56.

36. Anónimo. Sterilizing dental handpieces: results of a workshop on handpieces and other instruments in dentistry. I Am Dent Assoc 1992;123:44-47.

37. British Dental Association. Guidelines on infection control in dentistry: a practicioner's guide. London: BDA; 1993.

38. Nash KD. How infection control procedures are affecting dental practice today. J Am Dent Assoc 1992;123:67-73.

Manuscrito recibido el 3 de abril de 1996 y aceptado para publicación en versión revisada el 29 de octubre de 1996
ABSTRACT

\section{Dental care associated with an outbreak of HIV infection among dialysis patients}

An outbreak of 14 cases of human immunodeficiency virus (HIV) infection was discovered by chance in May 1993 among hemodyalisis patients at a university hospital in Bucaramanga, Colombia. The outbreak occurred in 1992. Stored sera were used to establish the probable period of infection (PPI) for 10 of the 14 cases. A nested casecontrol study was carried out to evaluate possible transmission mechanisms. The health care experience of each HIV-positive patient during that patient's PPI was compared to the experience of time-matched controls. Only invasive dental procedures were significantly associated with the risk of infection. Patients upon whom invasive dental procedures were performed during their PPIs had an average risk of HIV infection 8.15 times greater than comparable controls $(P=0,006)$, and seven out of nine cases of HIV infection with known PPIs in 1992 had an invasive dental procedure performed one to 6 months before seroconversion. None of the dental care personnel were found to be infected. Based on the available evidence, it seems most likely that the infection was transmitted from patient to patient by contaminated dental instruments. 